\title{
3D Body Scanning - Utilization of 3D Body Data for Garment and Footwear Design
}

\author{
Elfriede KIRCHDOERFER*, Angela MAHR-ERHARDT, Simone MORLOCK \\ Hohenstein Institut fuer Textile Innovationen, Germany
}

\begin{abstract}
As results of different measurement campaigns with 3D Body scanners, additional to the body measurements, the 3D scan data includes very valuable possibilities of utilisation for garment development and footwear design. Since 1999 a large database of individual 3D body and leg scans of female and male persons aged from 6 to 80 years are available at the Hohenstein Institute. This 3D scan data was the fundamental basis for several research projects. The data was used to develop body measurement tables for garments and foot wear, to generate average virtual body models and to extract geometrical body information for optimisation of garment pattern construction.
\end{abstract}

Keywords: anthropometric studies, anthropometrical measurements, size charts, 3D shape analysis, virtual body models, morphotypes, 2D and 3D pattern development

\section{Introduction}

In the past the intention to use 3D body scan data for the apparel industry was prior-ranking to extract body measurements to develop size charts $[1,2]$. Other very valuable 3D information such as 3D body shape, body surfaces etc. were only used in a small amount i.e. for visualization of size specific body morphologies and extraction of a small range of 3D body parameters to optimize pattern construction and fit of garments [1, 2]. At the first glance it seams a very expensive technology is used for collection of body measurements which could as well measured by tape. But the advantage of body scanning is to get more precise measurements without personal influences and the possibility to have a 3D digital twin of the scanned person available for future analysis.

Within the last decade with increasing of the engineering development of 3D software for garment simulation and pattern design, the utilization of 3D scan data became more important. For realistic virtual fit simulation avatars are required which represent real human body forms $[3,4,5,6,7,8]$. Correlations between body surface and their flattened geometry are required to integrate shape data into the pattern construction process to optimize fit of the garments.

During several research projects 3D body data has been used to extract geometric information for specific body parts and to generate average 3D body models or to extract relevant information about the body surface and its transformation into 2D plain. In the following different utilization of 3D body scan data will be demonstrated by presenting four different projects $[9,10,11,12]$.

\section{3D body information to extract morphological characteristics}

Additional to contoured and straight measurements measured on the body surface, body diameters in bust, under bust, waist and hip level provide a lot of information about body form and cross section in the above mentioned regions (see figure 1). Changing of the proportions between front and side diameter indicates alterations of the body shape. Using front diameters in combination of the shoulder width straight very exact data are available to define the development of the body shapes in front view with growing body volume.

Information to the posture are to extract out of measures which define the shape of the back profile. To a fictive vertical line the horizontal distances to the outer edge of the body shape in profile at neck, bust, waist and hip level were measured. In correlation to the vertical distances of the measuring positions significant features for each individual body posture are available. With the measurements of the back profile the derivation of average body posture for specific target groups is possible (see figure 2).

* e.kirchdoerfer@hohenstein.de; ++49 7143271 306; www.hohenstein.de 


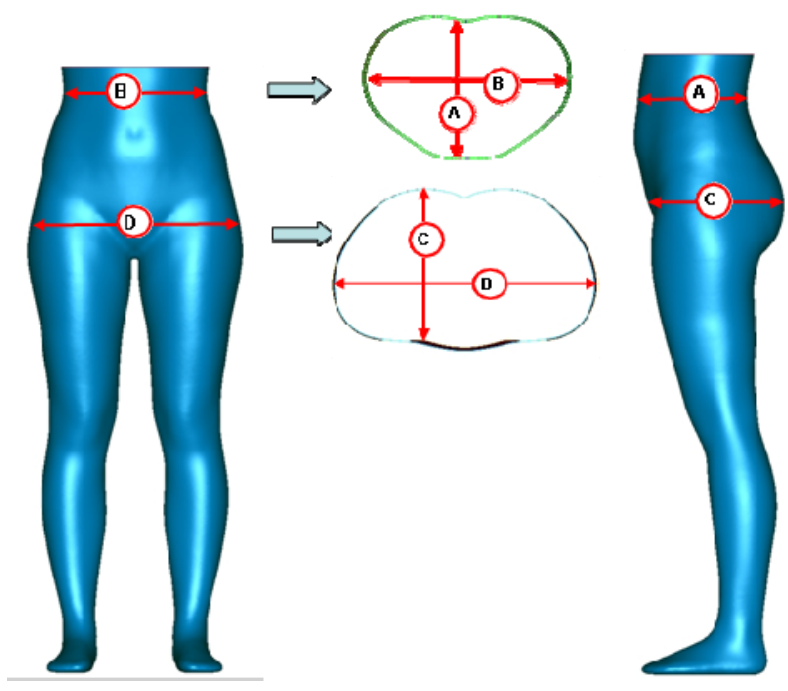

Figure 1: Body diameters at waist and hip level

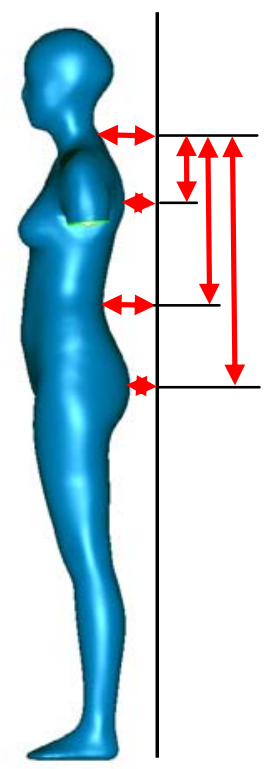

Figure 2: Measurements for posture definition

Besides the above mentioned measurements the whole body geometry is an essential base to improve pattern generation for different types of garments. Particular the body surface can act as provider of geometrical information to extract construction details are so far has to be derived by empirical methods. By transformation of the 3D body surface into a 2D plane, influences caused by size and shape of specific body morphologies could be identified (see figure 3).

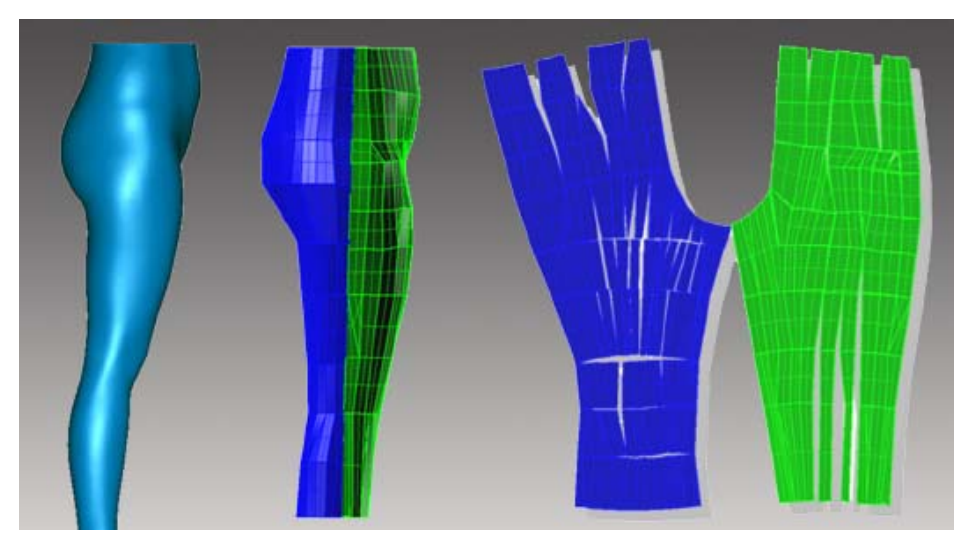

Figure 3: Transformation of 3D body surface into 2D plane 


\section{Utilization of 3D body data for garment and footwear design}

Based on examples of four different research projects, the utilization of 3D body data for garment and footwear design shall be demonstrated.

\subsection{Leg scanning for developing measurement charts and shoe lasts}

$3 \mathrm{D}$ whole body scanning is not accurate enough to derive required information for foot wear. Therefore more than 5.000 pairs of legs - male and female persons aged form 16 to 75 years - were collected by a specific leg scanner [9]. Within this research project the scan data was used to extract very detailed measurements of female and male legs from root base to knee level. Out of the measurement database size chart s were evaluated for shoe sizes, for socks and stockings. Based on the 3D leg geometry information for development of best fitting shoe lasts were identified (figure 4). The 3D scan data had also build the basis for 3D grading of the shoe lasts for different sizes and foot proportions. For extraction of significant differentiation based on the gender average leg models were generated (see figure 5).

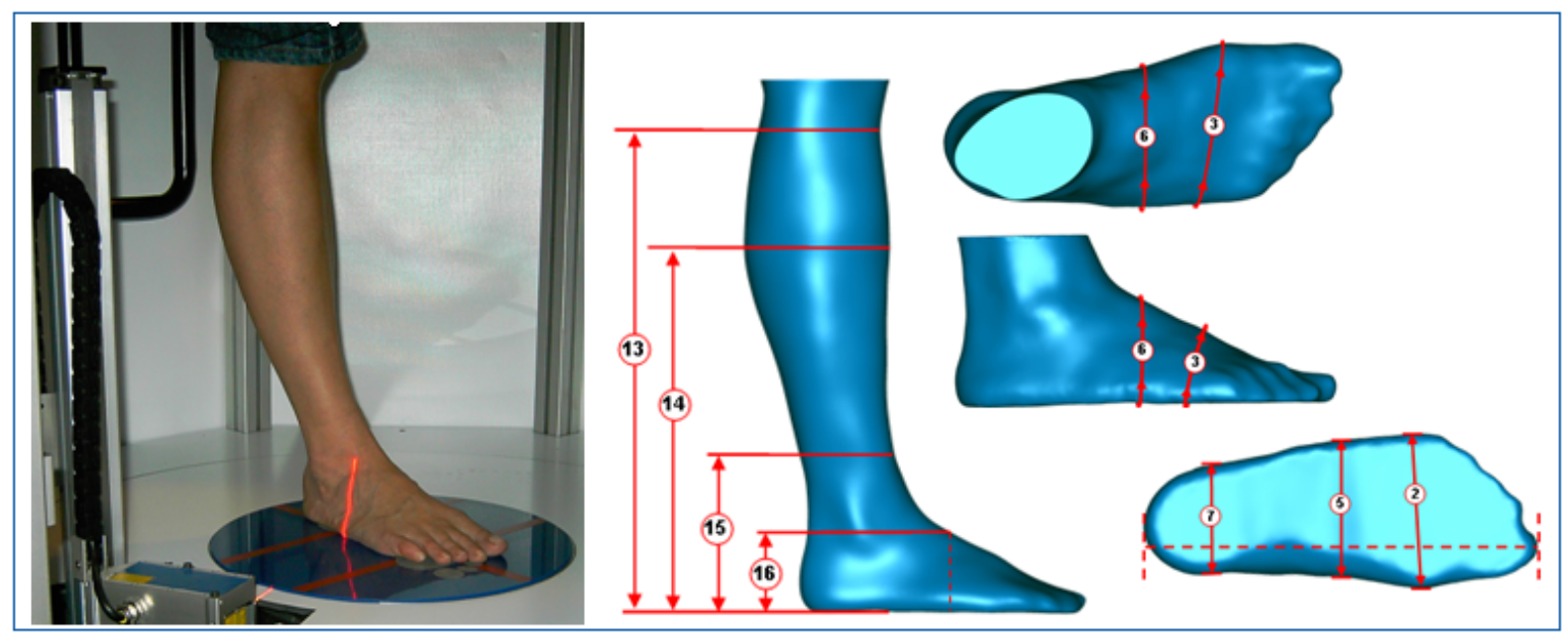

Figure 4: Detailed measurements of female and male legs from root base to knee level

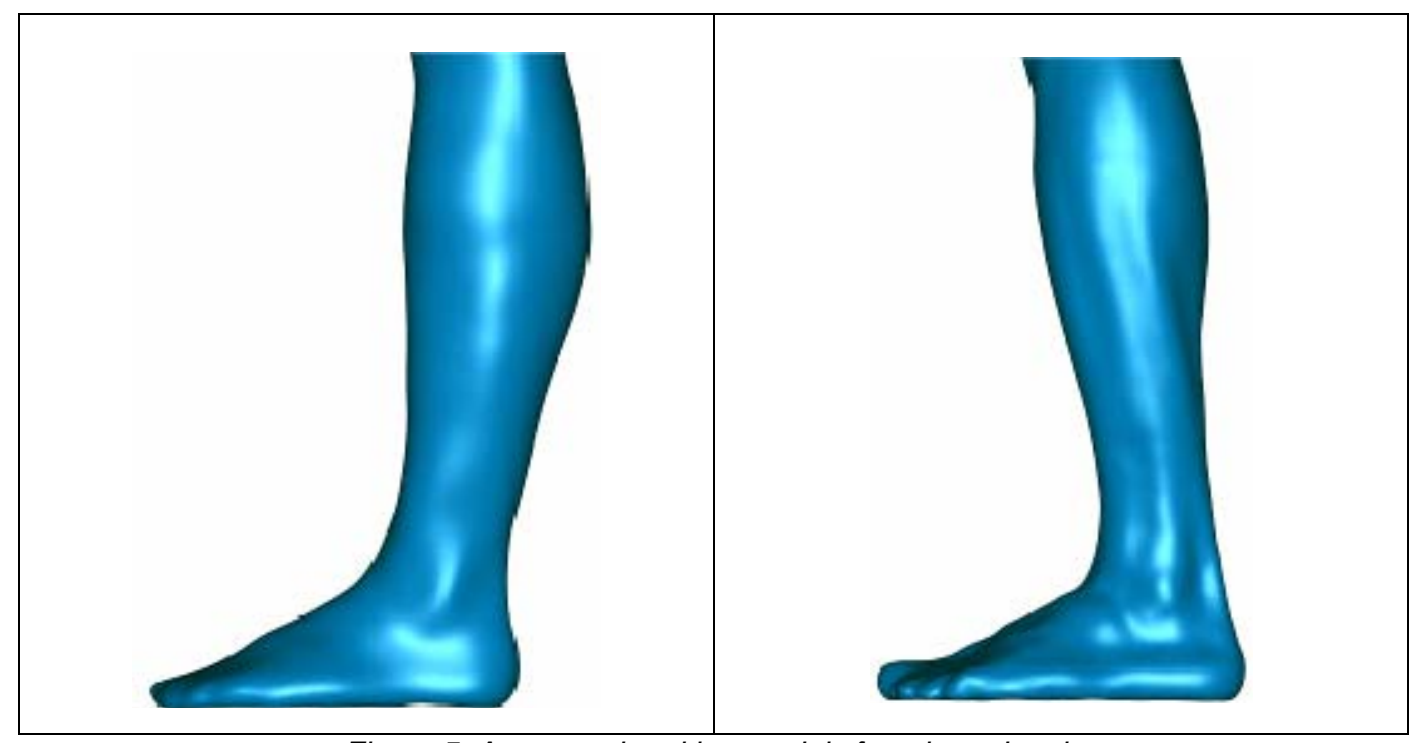

Figure 5: Average virtual leg models female and male 


\subsection{Development of morphology of big sizes - females}

The project was aimed to the special target group of female big sizes [10]. The usage of 3D body data was mainly focused to extract body measurements and to analyze the human body shape development caused by increasing of body volume and weight in order to identify size and age specific body morphologies. Out of the identified body morphologies virtual and physical body models were generated (see figure 6).

Body diameters in front and side view of the scanned persons were used as basis first to define the different morphotypes and second to extract the percentage distribution of the identified body forms. In the first step the main body form - A, H or V - was determined based on the correlation between shoulder width straight and the front diameter in hip level. Together with the body shape in waist area the final body shape is defined. The definition of the waist body shape is based on the proportions of the front diameter at bust, hip and waist level. Two different waist shapes were identified: $X$ - and $O$-waist shape. If the waist diameter of the person is bigger than the calculated waist diameter of the basis body shapes $\mathrm{A}, \mathrm{H}$, or $\mathrm{V}$, the waist body shape of the person will be an $\mathrm{O}$. If the waist diameter is smaller than the calculated one, the waist body shape of the person has to be defined as $\mathrm{X}$.

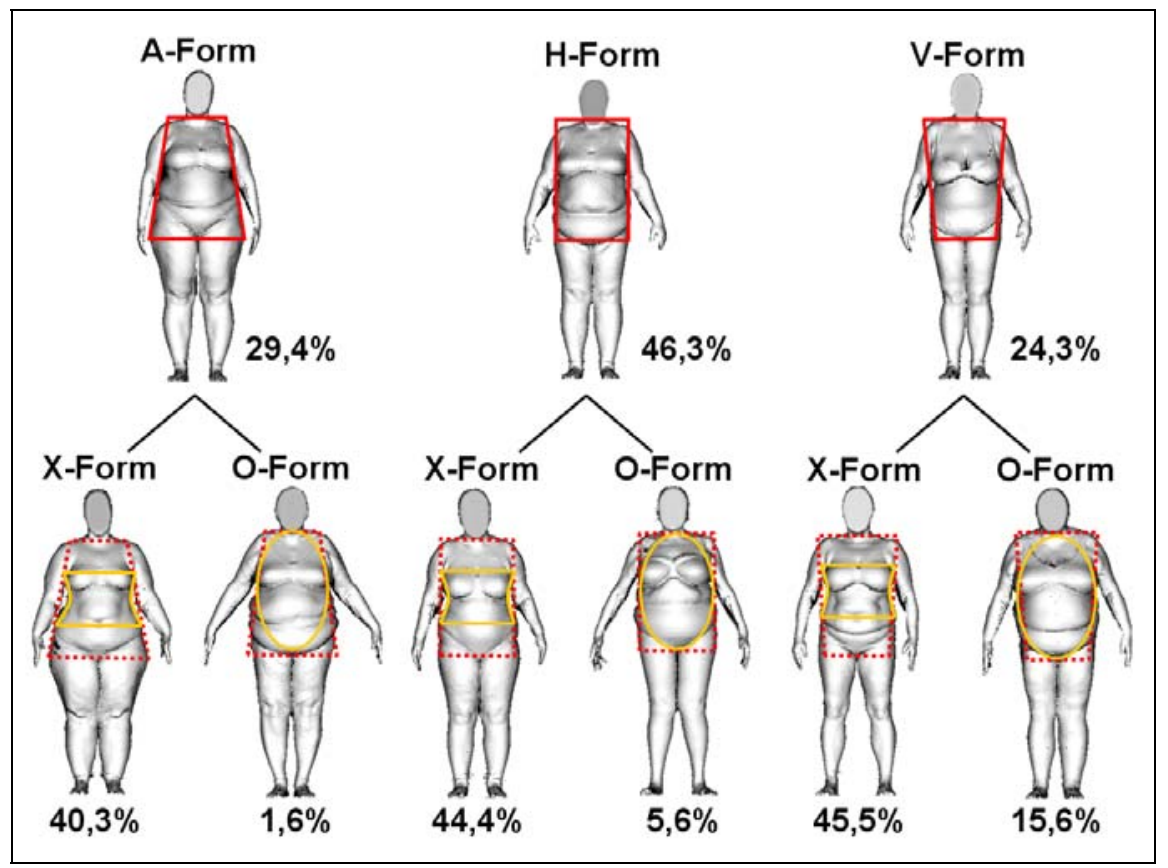

Figure 6: Identified body morphologies virtual and physical body models

\subsection{Generation of average virtual body models}

Aim of another project [11] was the development of average virtual body models which represent relating to dimensions, body shape and posture the majority of specific garment sizes (see figure 7). For realization a process was developed to generate realistic and replicable results for each size. The $3 \mathrm{D}$ body geometry builds the basis for generation of average virtual body models. Based on scan data of persons which fit with their basic measurements and their specific body features into the specific size the average body will be developed. The development happens in four steps: Development of the average torso, development of the average legs, development of the average arms and joining of the three parts. The process to develop average body parts includes following global working steps:

1. Copy and mirror the single body data. (This work is required to eliminate the influence of asymmetry between the left and right side of the body).

2. Adjustment of the single body geometry to reach a most similar posture for the whole data set.

3. Generation of the average body parts.

4. Combination of the separate developed body parts 


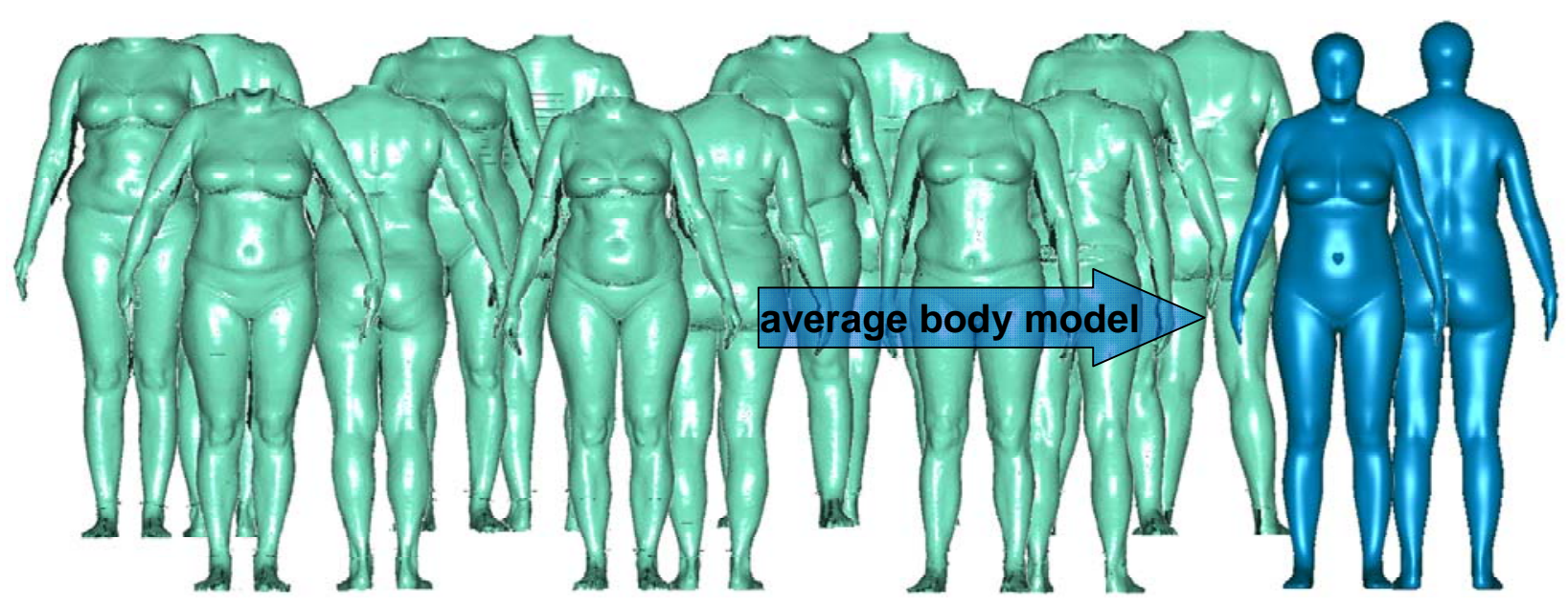

Figure 7: Average virtual body model - Size 46

\subsection{Extraction of geometric pattern information based on the body surface}

In a further research project based on 3D scan data, geometric information of body surfaces was extracted [12]. The objectives were to analyze 3D body data and its geometry to extract form information which could replace empirical construction elements of the current 2D pattern development methods for female trousers. To reach the aimed objective, basis for this project were individual 3D body data as well as average virtual body models for the different sizes.

To extract the relevant information about size and form specific influences into the pattern geometry, the body surface had to be transformed into 2D plains. By construction of 3D NURBS plains on the polygon meshes two results are reached: first the idealization of the body shape according to the influence of the specific garment and second the basis is created for 2D transformation of the body surface (see figure 8).
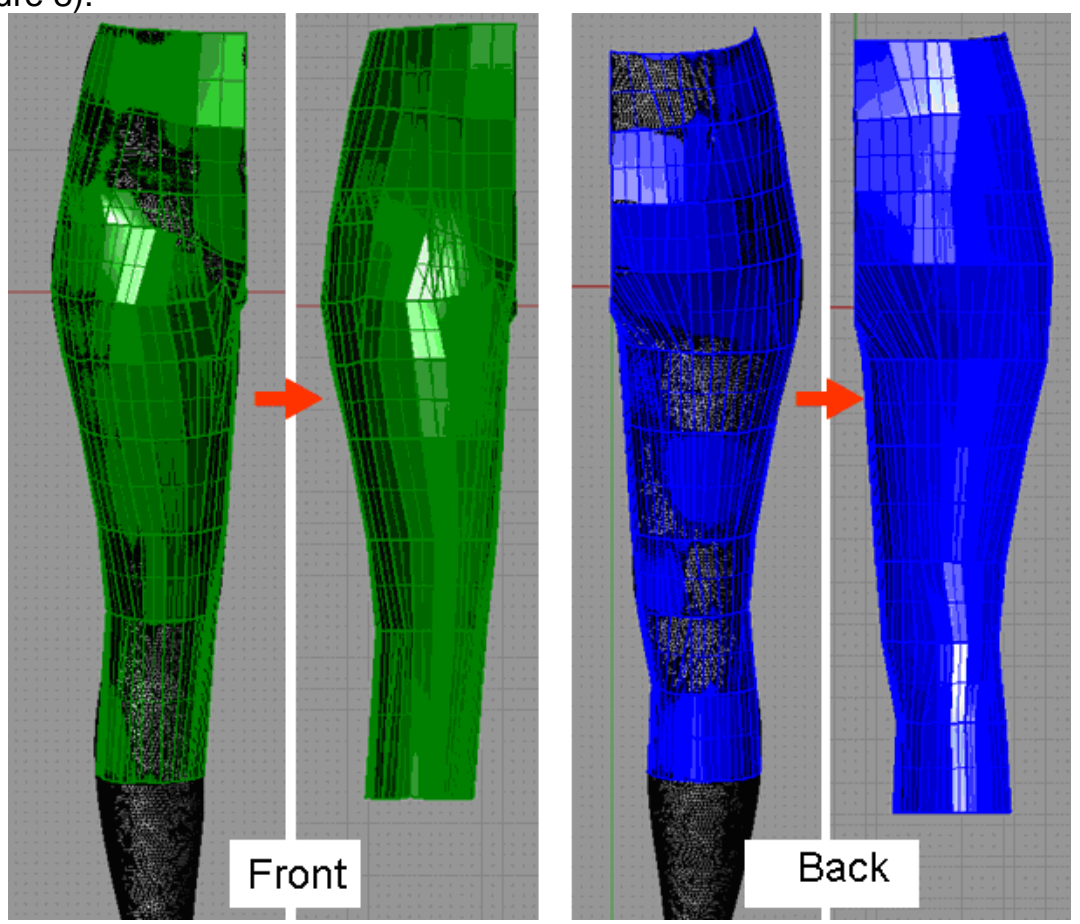

Figure 8: Extraction of geometric pattern information based on the body surface

After the flattening process the single pieces of the transformed body surface are to rearrange according to the aimed pattern geometry (see figure 9). 


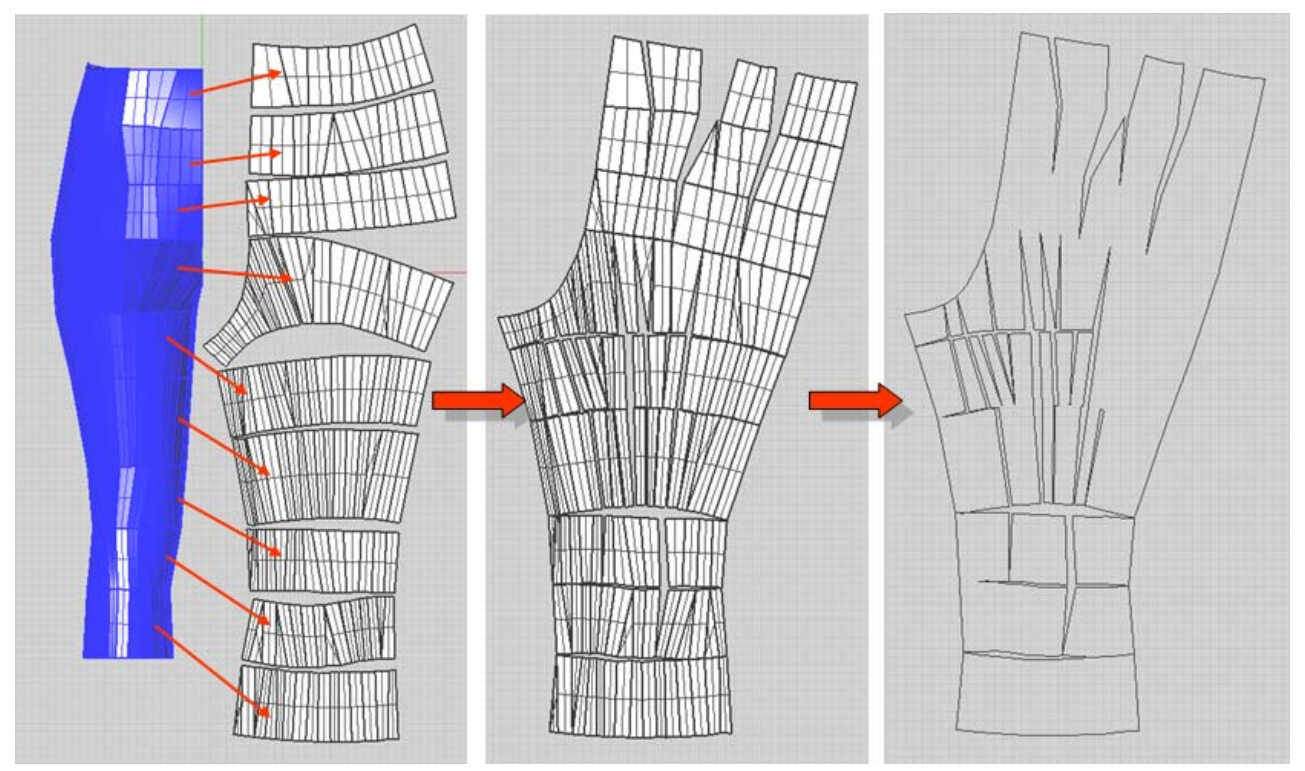

Figure 9: Rearranging of the flattened body surface

The transformed body surface contains a lot of information about the body shape, measurement position, body curves etc. (see figure 10). The comparison between measurement position at the body and the pattern shows that there are some differences. By translating extracted geometric information into the pattern development systems an increasing of optimized garment fit can be reached.

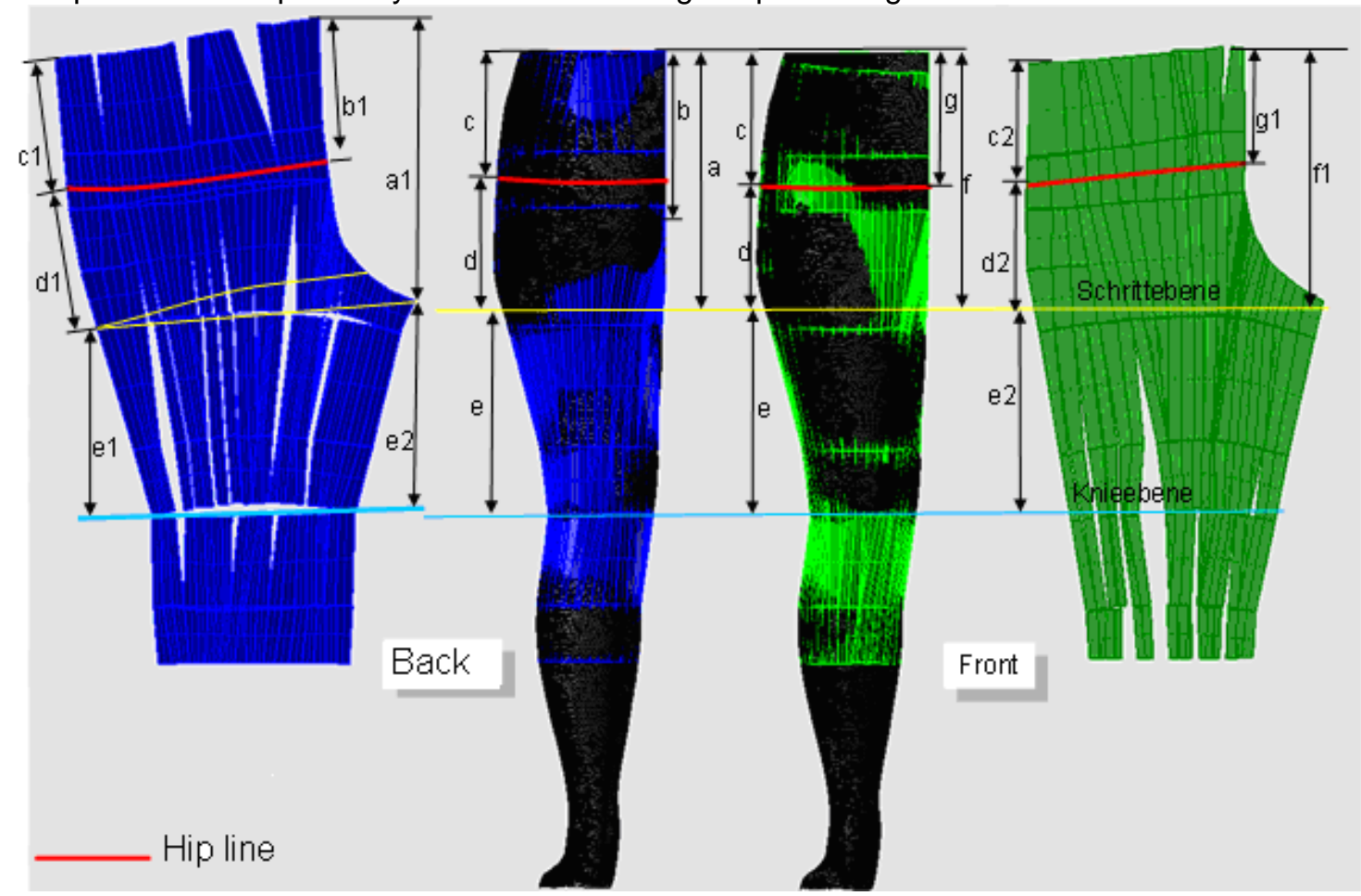

Figure 10: Comparison of body- and pattern measurements

The integration of the body diameter at hip level in body profile is very important to reach optimal fit. But to transform the 3D body dimension into the flat pattern is very complex. The diameter measured at the 3D body correlates in a high degree to the pattern dimension only if the front and back patterns are combined at the inner leg seam (see figure 10). This operation has been simulated by using the upper leg geometry to extract the angle "b" (see figure 11). This could be derived by transformation of the mathematical function for calculation of surface elements of a truncated cone according to thigh girth, knee circumference and the length of the upper leg from crotch to knee (see figure 12). 


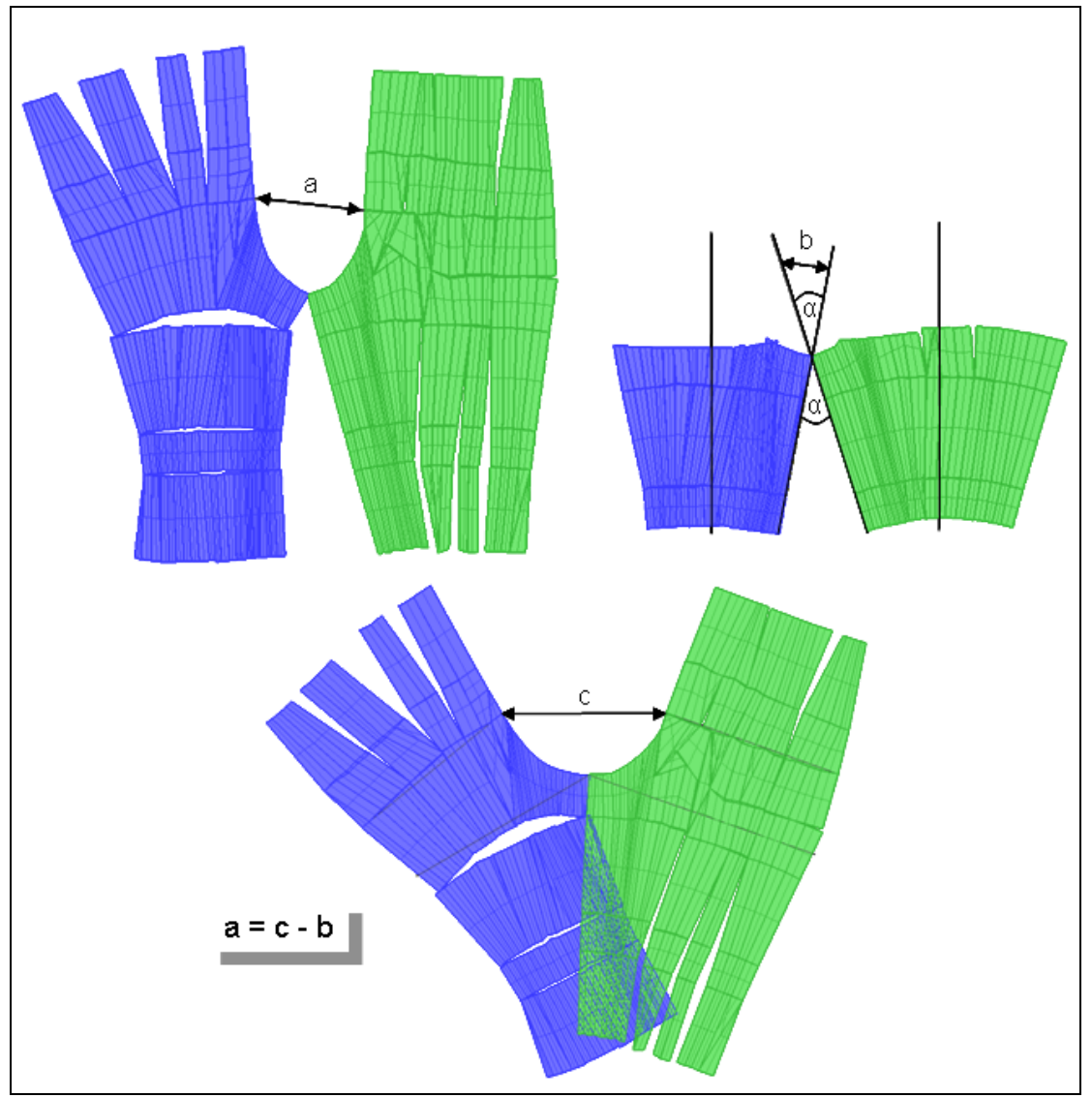

Figure 11: Transformation of hip diameter into pattern geometry

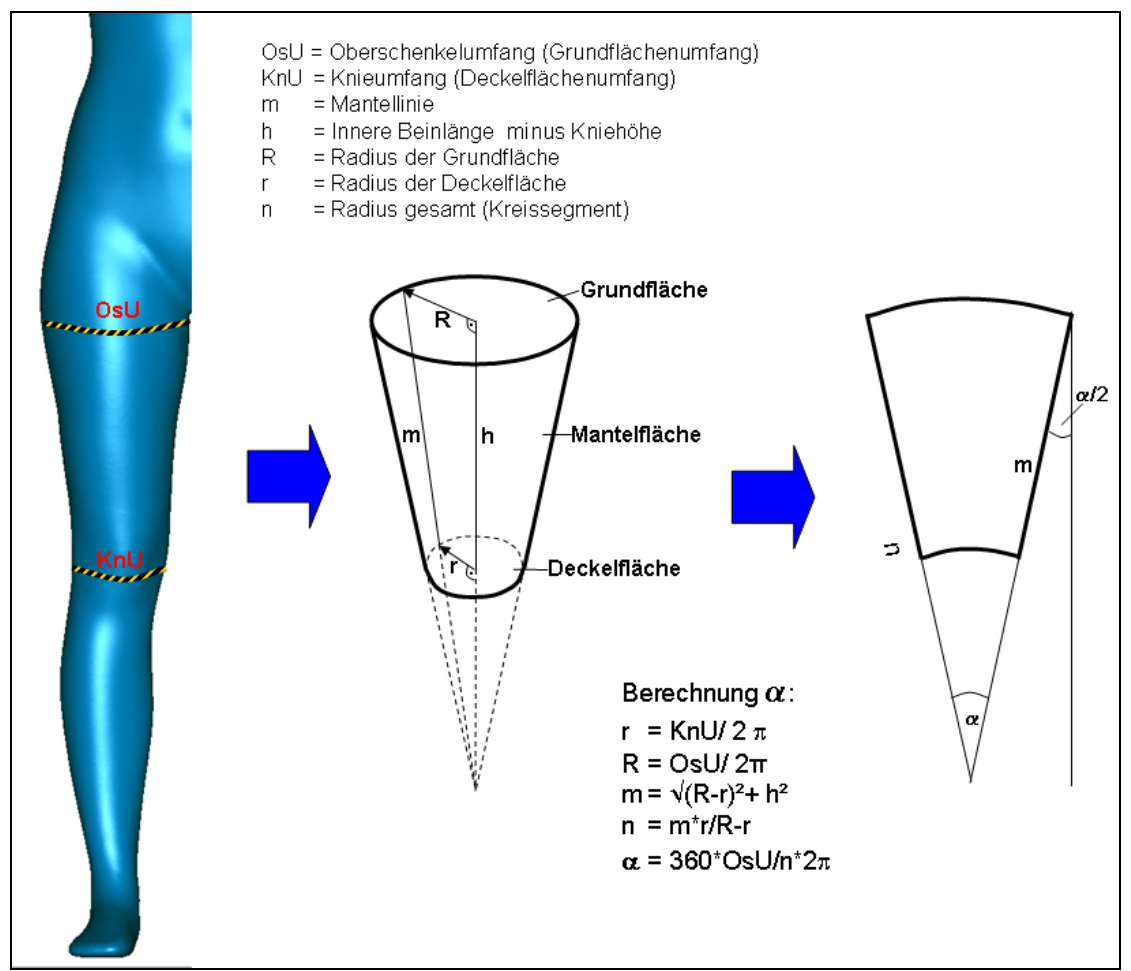

Figure 12: Transformation of the mathematical formula for a truncated cone 


\section{References}

1. Kirchdörfer, E.; Mahr-Erhardt, A.; Scheck, H.: Körpermaße, Marktanteile und Konstruktionsgrundlagen für Miederbekleidung. Bekleidungstechnische Schriftenreihe der Forschungsgemeinschaft Bekleidungsindustrie, Band 143, Köln, (2001)

2. Kirchdörfer, E.; Mahr-Erhardt, A.: Oberbekleidung für Frauen über 60 Jahre - Körperdimensionen, Größenverteilung, Schnittkonstruktion. Bekleidungstechnische Schriftenreihe der Forschungsgemeinschaft Bekleidungsindustrie, Band 156, Köln, 2003

3. nn. BMBF Projekt: Virtual-Try-on - Von der "Virtuellen Anprobe" zum Maßschnitt (2004)

4. Optitex. http://www.optitex.com/ , (2009)

5. V-Sticher. http://www.browzwear.com/, (2009)

6. Fashionizer. http://www.miralab.unige.ch/, (2009)

7. Vidya. http://www.assyst-intl.com/assyst/, (2009)

8. I-Designer. http://www.i-designer-web.com/, (2009)

9. nn. Entwicklung innovativer Gradierverfahren für die Schuh- \& Strumpfwarenindustrie, Abschlussbericht zum AiF Vorhaben Nr.14993 BG; (2009)

10. Morlock, S., Wendt, E., Krzywinski, S., Kirchdörfer, E., Rödel, H., Rupp, M., Grundsatzuntersuchung zur Konstruktion passformgerechter Bekleidung für Frauen mit starken Figuren, Schlussbericht zum AiF-Vorhaben Nr. 15144 BG, (2009)

11. Hohenstein Institute, Human Solutions GmbH;. http://www.sizegermany.de/, (2009)

12. Kirchdoerfer, E. et.al.: Entwicklung einer automatischen, körperkonformen Konstruktionssystematik für Oberbekleidung auf Basis von 3D Körper Informationen, Schlussbericht zum AiF Vorhaben Nr.15605 N, (2011) 